\title{
Welcome message from the Editor-in-Chief
}

\author{
Peng Liu \\ College of Information Sciences and Technology, Pennsylvania State University, University Park, PA 16802, USA
}

\begin{abstract}
Received on 7 June 2011
Copyright $\odot 2011 \mathrm{Liu}$, licensed to ICST. This is an open access article distributed under the terms of the Creative Commons Attribution licence (http://creativecommons.org/licences/by/3.0/), which permits unlimited use, distribution and reproduction in any medium so long as the original work is properly cited.
\end{abstract}

doi: 10.4108/trans.sesa.2011.el

On behalf of the Editorial Board of ICST Transactions on Security and Safety (ISSN\# 2032-9393), I welcome you to the inaugural issue of this international archived journal.

Creation of this new journal is not only motivated by the increasing importance of security and safety, but also by the observation that although there exist a good number of journals in the field, researchers doing interdisciplinary researches and exploring out-of-the-box ideas still have difficulty finding a suitable journal to publish their findings.

The goal of this journal is threefold: (i) to provide a dedicated venue for interdisciplinary researches and out-of-the-box ideas; (ii) to help form a community for interdisciplinary security researches; and (iii) to help this community improve academic rigor.

The scope of this journal is by design interdisciplinary, covering both the natural science aspects and the social/ behavior science aspects of security and safety. The expertise of the Editorial Board includes and is not limited to online safety and privacy, homeland security and risk, computer and network security, and economics of information security.

Besides academic research papers, which will be rigorously reviewed by experts from the community, we will also put in major effort to solicit and publish position papers written by leading practitioners, security professionals, and policy makers. These position papers will help shorten the 'distance' between academic research and the real world.

In this inaugural issue, Professor Heng Xu from The Pennsylvania State University, serving as the Guest Editor, and the authors have put together an exciting special issue on 'online safety and privacy'. I want to thank Professor Xu and the authors for their great effort. Details of this special issue are in the Editorial written by Professor Xu.

Regarding the following issues of this journal, besides issues formed by regular submissions, a special issue on Cyber Situation Awareness has been planned.

Email: pliu@ist.psu.edu
I and the ICST Transactions on Security and Safety team hope you have a rich and enjoyable experience with this issue and the following issues of this journal.

\section{About the Editor-in-Chief}

Peng Liu received his BS and MS degrees from the University of Science and Technology of China, and his $\mathrm{PhD}$ degree from George Mason University in 1999. Dr Liu is a full professor of Information Sciences and Technology, director of the Center for Cyber-Security, Information Privacy, and Trust, and director of the Cyber Security Lab at Penn State. His research interests are in all areas of computer and network security, and he has done substantial research in software security.

$\mathrm{He}$ is the program co-chair of ACM ASIACCS 2010, SECURECOMM 2008, and DBSEC 2006, General Chair of DBSEC 2010 and SECURECOMM 2009, and the founding program co-chair of the ACM Workshop on Survivable and Self-Regenerative Systems. He is a program committee member of over 80 international conferences, including CCS, INFOCOM, and WWW. $\mathrm{He}$ is a referee for over 20 journals, including ACM Transactions on Information and Systems Security and IEEE Transactions on Dependable and Secure Computing. $\mathrm{He}$ is the Editor-in-Chief of ICST Transactions on Security and Safety. He is and has served on several editorial boards, including Wiley Security and Communication Networks Journal and Elsevier Computers \& Security Journal.

He has published a book and over 180 refereed technical papers. His research has been sponsored by DARPA, NSF, AFOSR, ARO, DHS, DOE, AFRL, NSA, TTC, CISCO, and HP (In total, he has secured over 14 million US dollars as a PI or Co-PI). Dr Liu is a recipient of the DOE Early Career Principle Investigator Award. He has co-led the effort to make Penn State a NSA-certified National Center of Excellence in Information Assurance Research. More information about him can be found at http://s2.ist.psu.edu. 